\title{
The evolving treatment paradigm for BRAF V600 mutant colorectal cancer
}

\author{
Jeremy D. Kratz ${ }^{1}$, Dustin A. Deming ${ }^{1,2,3}$ \\ ${ }^{1}$ Department of Medicine, University of Wisconsin School of Medicine and Public Health, Madison, WI, USA; ${ }^{2}$ Department of Oncology, McArdle \\ Laboratory for Cancer Research, University of Wisconsin School of Medicine and Public Health, Madison, WI, USA; ${ }^{3}$ University of Wisconsin \\ Carbone Cancer Center, Madison, WI, USA \\ Correspondence to: Dustin A. Deming, MD. 6507 Wisconsin Institutes for Medical Research, 1111 Highland Ave, Madison, WI 53705, USA. \\ Email: ddeming@medicine.wisc.edu. \\ Provenance: This is an invited article commissioned by the Academic Editor, Dr. Chun-Dong Zhang, PhD (Department of Gastrointestinal Surgery, \\ Graduate School of Medicine, University of Tokyo, Tokyo, Japan). \\ Comment on: Van Cutsem E, Huijberts S, Grothey A, et al. Binimetinib, Encorafenib, and Cetuximab Triplet Therapy for Patients With BRAF \\ V600E-Mutant Metastatic Colorectal Cancer: Safety Lead-In Results From the Phase III BEACON Colorectal Cancer Study. J Clin Oncol \\ 2019;37:1460-9.
}

Submitted Nov 01, 2019. Accepted for publication Dec 10, 2019.

doi: $10.21037 /$ atm.2019.12.61

View this article at: http://dx.doi.org/10.21037/atm.2019.12.61

\section{Introduction}

Metastatic colorectal cancer (CRC) is now being better understood as a collection of diseases with each distinguished by distinct molecular profiles. BRAF mutant cancers are an important CRC subtype composing about $10 \%$ of metastatic CRCs. Of those with a mutation in the BRAF gene, $80 \%$ possess the V600E mutation (1). This alteration results in a constitutively active kinase which activates the downstream MEK/ERK signaling pathway. $\mathrm{BRAF}^{\mathrm{V} 600}$ mutant cancers are more commonly on the right side of the colon and have a higher prevalence in women (2). These cancers have a preponderance for extensive nodal disease, peritoneal disease, and a very poor prognosis, with a median survival for patients with $B R A F^{V 600}$ mutant metastatic CRC of less than 2 years (3-5).

$B R A F^{V 600}$ mutations are associated with relative chemotherapy resistance and rapid progression in the setting of chemotherapy resistance. For patients with appropriate performance status, combination cytotoxic chemotherapy remains the consensus standard of care. FOLFOX and FOLFIRI regimens are commonly utilized (6). $B R A F$ mutations are associated with a lack of survival benefit from the anti-epidermal growth factor receptor (EGFR) inhibitors, cetuximab and panitumumab (5,7-9), but BRAF mutant cancers can still benefit from bevacizumab (10). Given the poor prognosis and decreased performance status, many patients with $B R A F$ mutant CRC are not candidates for second-line therapy. This has led to interest in using FOLFOXIRI and bevacizumab in the first-line setting. The TRIBE study investigated this regimen in a post-hoc analysis of small cohort $(n=28)$ of patients demonstrating an improvement in overall survival from 10.7 to 19 months when compared to FOLFIRI and bevacizumab, although not statistically significant with hazard ratio (HR) $0.54(0.24,1.20)$ (4).

In contrast to $B R A F$ mutant melanoma, $B R A F$ mutant CRCs are largely resistant to single agent BRAF targeted therapies (11-13). Preclinical studies have revealed feedback signaling results in increased EGFR signaling mediated downstream by direct activation of CRAF or by the transactivation of BRAF-CRAF heterodimers (14). This has led to combination EGFR and BRAF inhibitor regimens with improved response rates (15-17). The combination of vemurafenib, cetuximab and irinotecan demonstrated a response rate (RR) of $16 \%$ versus $0 \%$ with standard chemotherapy and an improvement in median progression free survival (PFS) of 4.4 versus 2.0 months (HR $0.42, \mathrm{P}<0.001$ ) in patients with treatment refractory metastatic $B R A F^{V 600}$ mutant CRC (18). Most recently triplet combinations targeting EGFR, BRAF, and MEK have 
Table 1 Clinical trials of targeted therapy for BRAF mutant metastatic colorectal cancer

\begin{tabular}{|c|c|c|c|c|c|c|c|c|}
\hline Therapeutic targets & Study & Phase & Inclusion criteria & Therapy & $\mathrm{n}$ & $\mathrm{RR}(\%)$ & $\begin{array}{l}\text { PFS } \\
\text { (mos) }\end{array}$ & $\begin{array}{c}\text { OS } \\
\text { (mos) }\end{array}$ \\
\hline \multicolumn{9}{|l|}{ Triplet therapy } \\
\hline \multirow[t]{2}{*}{$\begin{array}{l}\text { BRAF/MEK/EGFR } \\
\text { inhibition }\end{array}$} & BEACON CRC & III & $\geq 1$ lines of systemic therapy & $\begin{array}{l}\text { Encorafenib, binimetinib, } \\
\text { cetuximab }\end{array}$ & 224 & 26 & 4.3 & 9.0 \\
\hline & NCT01750918 & I & Any line of therapy & $\begin{array}{l}\text { Dabrafenib, trametinib, } \\
\text { panitumumab }\end{array}$ & 91 & 21 & 4.2 & $\mathrm{~N} / \mathrm{A}$ \\
\hline $\begin{array}{l}\text { BRAF/PIK3CA/EGFR } \\
\text { inhibition }\end{array}$ & NCT01719380 & $\mathrm{lb} / \mathrm{II}$ & $\geq 1$ lines of systemic therapy & $\begin{array}{l}\text { Encorafenib, alpelisib, } \\
\text { cetuximab }\end{array}$ & 52 & 27 & 5.4 & 13.1 \\
\hline $\begin{array}{l}\text { BRAF/EGFR Inhibition } \\
\text { with chemotherapy }\end{array}$ & SWOG1406 & II & $\geq 1$ lines of systemic therapy & $\begin{array}{l}\text { Vemurafenib, cetuximab, } \\
\text { irinotecan }\end{array}$ & 49 & 16 & 4.4 & 9.6 \\
\hline \multirow{3}{*}{ BRAF/EGFR inhibition } & NCT01524978 & $\begin{array}{l}\text { II, } \\
\text { baske }\end{array}$ & Any line of therapy & Vemurafenib, cetuximab & 27 & 4 & 4.5 & 9.3 \\
\hline & NCT01750918 & I & Any line of therapy & Dabrafenib, panitumumab & 20 & 10 & 3.5 & 8.4 \\
\hline & NCT01791309 & I & Any line of therapy & Vemurafenib, panitumumab & 15 & 13 & 3.2 & 7.6 \\
\hline BRAF/MEK inhibition & NCT01072175 & I/II & Any line of therapy & Dabrafenib, trametinib & 43 & 12 & 3.5 & N/A \\
\hline \multirow[t]{2}{*}{$\begin{array}{l}\text { Non-BRAF therapy } \\
\text { (control arms) }\end{array}$} & BEACON CRC & III & $\geq 1$ lines of systemic therapy & $\begin{array}{l}\text { Irinotecan, cetuximab or } \\
\text { FOLFIRI, cetuximab }\end{array}$ & 107 & 4 & 1.5 & 5.4 \\
\hline & SWOG1406 & II & $\geq 1$ lines of systemic therapy & Irinotecan, cetuximab & 50 & 4 & 2.0 & 5.9 \\
\hline
\end{tabular}

been investigated to enhance the efficacy of this treatment strategy $(16,19)$ (Table 1).

\section{Phase III BEACON Colorectal Cancer Study}

Dr. Van Cutsem, et al., published the results of the safety lead in of the phase III BEACON Colorectal Cancer Study examining the combination of binimetinib, encorafenib and cetuximab (19). This manuscript detailed the results of the first 30 patients enrolled in this clinical trial examining the use of this regimen for patients with $B R A F^{V 600 E}$ mutant metastatic CRC in the treatment refractory setting. The primary endpoint of the safety lead-in was to determine the safety and tolerability of this triplet regimen dosed at encorafenib $300 \mathrm{mg}$ every day, binimetinib $45 \mathrm{mg}$ twice a day and cetuximab $400 \mathrm{mg} / \mathrm{m}^{2}$ followed by $250 \mathrm{mg} / \mathrm{m}^{2}$ intravenously weekly in 28-day cycles. Dose limiting toxicities were observed in 5 of the 30 patients, including serous retinopathy, reversible decreased left ventricular ejection fraction, and cetuximab-related infusion reactions. The most common grade 3/4 adverse events included fatigue, anemia, increased creatine phosphokinase, increased AST, and urinary tract infections. Overall, this regimen was deemed tolerable. Interestingly, diarrhea and acneiform rash were less severe than reported prior studies of MEK and EGFR inhibition alone (16,20). Preliminary clinical efficacy was also evaluated in this cohort of patients identifying a RR of $48 \%$ and median PFS of 8.0 months.

Most recently, the prespecified interim analysis of the Phase III BEACON Colorectal Cancer Study was reported (21). This trial enrolled 665 patients with $\mathrm{BRAF}^{\mathrm{V} 600 \mathrm{E}}$ evenly across three cohorts, including the triplet regimen of encorafenib, binimetinib and cetuximab; the doublet regimen of encorafenib and cetuximab; and a control cohort where patients could receive either cetuximab and irinotecan or cetuximab with FOLFIRI 
(folinic acid, 5-fluorouracil, and irinotecan). The primary endpoints were overall survival (OS) and RR between the triplet regimen and the control group. The statistical analysis plan was not designed to compare the triplet and doublet regimens. The three cohorts were similar at baseline with the majority of patients being treated in the second-line setting (66\%). At the time of interim analysis, the median duration of survival follow-up is 7.8 months. The median OS for the control group was 5.4 months, 8.4 months in the doublet therapy group, and 9.0 months with the triplet therapy (comparing the triplet therapy vs. control; HR 0.52; $\mathrm{P}<0.001)$. A blinded independent central review assessed $R R$ in this analysis for the first 331 patients who underwent randomization. The RR in the control arm was $2 \%$ compared to $20 \%$ with the doublet therapy and $26 \%$ in the triplet therapy group (comparing control vs. triplet therapy; $\mathrm{P}<0.001$ ). Of note, a substantial portion of the patients were not able to be evaluated for response with the majority of these patients being those with clinical progression or who discontinued therapy due to adverse events (15.4\%). Median PFS, by central review, was 1.5 months in the control group, 4.2 months in the doublet therapy group, and 4.3 months with triplet therapy. Interestingly, the percent of patients with a response who had a duration of response $\geq 6$ months was greater in the doublet therapy (43\%) than with the triplet regimen (24\%). Toxicities were similar to that seen in the lead-in phase, however notable improvements in toxicities were seen with the doublet compared to the triplet regimen including diarrhea ( $62 \%$ vs. $33 \%)$, acneiform dermatitis ( $49 \%$ vs. $29 \%)$, nausea ( $45 \%$ vs. $34 \%$ ), and vomiting (38\% vs. $21 \%)$.

\section{Discussion}

Reduced cost next-generation sequencing has resulted in a dramatically improved understanding of CRC including distinct molecular subtypes. It is now standard of care to test the tumors of patients with metastatic CRC for KRAS/NRAS/BRAF mutations, HER2 amplification, and microsatellite instability/mismatch repair deficiency. These molecular alterations are both prognostic and predictive and aid in defining personalized treatment algorithms at diagnosis or first evidence of metastatic disease.

The phase III BEACON Colorectal Cancer Study provides a significant advance for those patients with $B R A F^{V 600}$ mutant metastatic CRC. It is now standard of care to treat patients with $B R A F^{V 600}$ mutant metastatic
CRC with the triplet regimen of encorafenib, binimetinib and cetuximab in the treatment refractory setting. It should also be considered reasonable to replace cetuximab with panitumumab if desired by the treating physician. This regimen should replace the use of an anti-EGFR therapy with chemotherapy or as part of the vemurafenib, irinotecan, and cetuximab regimen. It is notable how poorly the control group did with this prior standard therapy.

Of note, not all BRAF mutations have the same clinical impact with $\mathrm{BRAF}^{\mathrm{non}-\mathrm{V} 600}$ mutations ( $2 \%$ of CRCs) largely having a more indolent clinical course $(22,23)$. These mutations can result in different changes in the BRAF protein other than activating the kinase domain. For now, these regimens presented here should be restricted to those cancers with $B R A F^{V 600}$ mutations or at least alterations that are thought to result in a similar activation of the kinase domain.

Further follow-up is needed to assess the differences between the doublet and triplet regimens. The triplet regimen may result in greater treatment response, however this study was not powered to detect this difference and comes at the expense of greater toxicities. For now, the triplet regimen is preferred due to the response benefit. However, should a patient develop treatment related toxicities requiring dose modification, if these toxicities could in any way be attributable to the MEK inhibitor than would favor discontinuation of binimetinib, over dose reduction or delay of encorafenib or cetuximab. Additionally, for some patients with worse performance status or co-morbidities it would be reasonable to initiate therapy doublet regimen with the doublet regimen.

$B R A F^{V 600}$ mutations also occur in cancers with mismatch repair deficiency (dMMR). BRAF mutations can occur in tumors that develop through the serrated/methylated pathway, which are characterized by the $\mathrm{CpG}$ island methylator phenotype (CIMP) (24). CIMP-high tumors commonly possess epigenetic silencing of mutL homolog 1 (MLH1) resulting in dMMR. Those cancers with a $B R A F^{V 600}$ mutation and $\mathrm{d} M M \mathrm{R}$ have an intermediate prognosis between $B R A F^{V 600}$ mutant cancers without dMMR and BRAF wild-type cancers (24). The Checkmate-142 study recently demonstrated benefit for dMMR CRCs with single agent nivolumab and the combination of nivolumab and ipilimumab. Specifically for those $\mathrm{dMMR}$ cancers with BRAF mutations a RR of $25 \%$ was observed with nivolumab alone and a RR of $55 \%$ was observed with the combination of nivolumab and ipilimumab (25). Anti-PD1 therapies also provide the potential for a much longer duration 
of response, thus would prefer immunotherapy prior to targeted therapy for patients with $B R A F^{V 600}$ mutant $\mathrm{d} M M \mathrm{R}$ cancers.

In summary, $B R A F^{V 600}$ mutant metastatic CRC is a distinct biological subtype with a poor prognosis and evolving treatment considerations. Cytotoxic chemotherapy in combination with anti-angiogenic therapy remains the standard of care first-line option. Future clinical studies are needed to assess the benefit of targeted therapies in the first-line setting to better understand which patients with $B R A F^{V 600}$ mutant CRC are most likely to benefit.

\section{Acknowledgments}

Funding: The authors thank the University of Wisconsin Biology of Aging and Age-Related Diseases T32 Training Grant \#5T32AG000213-28 for providing salary support for Dr. Kratz.

\section{Footnote}

Conflicts of Interest: Dr. Kratz has no conflicts of interest to declare. Dr. Deming has served on an advisory board for Array BioPharma.

Ethical Statement: The authors are accountable for all aspects of the work in ensuring that questions related to the accuracy or integrity of any part of the work are appropriately investigated and resolved.

\section{References}

1. Cancer Genome Atlas Network. Comprehensive molecular characterization of human colon and rectal cancer. Nature 2012;487:330-7.

2. Loupakis F, Moretto R, Aprile G, et al. Clinicopathological nomogram for predicting BRAF mutational status of metastatic colorectal cancer. Br J Cancer 2016;114:30-6.

3. Innocenti F, Ou FS, Zemla T, et al. Somatic DNA mutations, MSI status, mutational load (ML): Association with overall survival (OS) in patients (pts) with metastatic colorectal cancer (mCRC) of CALGB/SWOG 80405 (Alliance). American Society of Clinical Oncology, 2017.

4. Cremolini C, Loupakis F, Antoniotti C, et al. FOLFOXIRI plus bevacizumab versus FOLFIRI plus bevacizumab as first-line treatment of patients with metastatic colorectal cancer: updated overall survival and molecular subgroup analyses of the open-label, phase 3 TRIBE study. Lancet Oncol 2015;16:1306-15.

5. Bokemeyer C, Van Cutsem E, Rougier P, et al. Addition of cetuximab to chemotherapy as first-line treatment for KRAS wild-type metastatic colorectal cancer: pooled analysis of the CRYSTAL and OPUS randomised clinical trials. Eur J Cancer 2012;48:1466-75.

6. Benson AB, Venook AP, Al-Hawary MM, et al. NCCN guidelines insights: colon cancer, version 2.2018. J Natl Compr Canc Netw 2018;16:359-69.

7. Pietrantonio F, Petrelli F, Coinu A, et al. Predictive role of BRAF mutations in patients with advanced colorectal cancer receiving cetuximab and panitumumab: a metaanalysis. Eur J Cancer 2015;51:587-94.

8. Douillard JY, Oliner KS, Siena S, et al. PanitumumabFOLFOX4 treatment and RAS mutations in colorectal cancer. N Engl J Med 2013;369:1023-34.

9. Rowland A, Dias M, Wiese M, et al. Meta-analysis of BRAF mutation as a predictive biomarker of benefit from anti-EGFR monoclonal antibody therapy for RAS wild-type metastatic colorectal cancer. Br J Cancer 2015;112:1888-94.

10. Nakayama I, Shinozaki E, Matsushima T, et al. Retrospective study of RAS/PIK3CA/BRAF tumor mutations as predictors of response to first-line chemotherapy with bevacizumab in metastatic colorectal cancer patients. BMC Cancer 2017;17:38.

11. Kopetz S, Desai J, Chan E, et al. Phase II pilot study of vemurafenib in patients with metastatic BRAF-mutated colorectal cancer. J Clin Oncol 2015;33:4032-8.

12. Falchook GS, Long GV, Kurzrock R, et al. Dabrafenib in patients with melanoma, untreated brain metastases, and other solid tumours: a phase 1 dose-escalation trial. Lancet 2012;379:1893-901.

13. Gomez-Roca C, Delord J, Robert C, et al. 535P Encorafenib (LGX818), an oral BRAF inhibitor, in patients (pts) with BRAF V600E metastatic colorectal cancer (mCRC): results of dose expansion in an openlabel, phase 1 study. Ann Oncol 2014;25:iv182-iv3.

14. Corcoran RB, Ebi H, Turke AB, et al. EGFR-mediated reactivation of MAPK signaling contributes to insensitivity of BRAF-mutant colorectal cancers to RAF inhibition with vemurafenib. Cancer Discov 2012;2:227-35.

15. Hyman DM, Puzanov I, Subbiah V, et al. Vemurafenib in Multiple Nonmelanoma Cancers with BRAF V600 Mutations. N Engl J Med 2015;373:726-36.

16. Corcoran RB, Andre T, Atreya CE, et al. Combined BRAF, EGFR, and MEK Inhibition in Patients with 
BRAF(V600E)-Mutant Colorectal Cancer. Cancer Discov 2018;8:428-43.

17. van Geel RM, Tabernero J, Elez E, et al. A phase Ib doseescalation study of encorafenib and cetuximab with or without alpelisib in metastatic BRAF-mutant colorectal cancer. Cancer Discov 2017;7:610-9.

18. Kopetz S, McDonough SL, Morris VK, et al. Randomized trial of irinotecan and cetuximab with or without vemurafenib in BRAF-mutant metastatic colorectal cancer (SWOG 1406). American Society of Clinical Oncology, 2017.

19. Van Cutsem E, Cuyle PJ, Huijberts S, et al. BEACON CRC study safety lead-in (SLI) in patients with BRAF V600E metastatic colorectal cancer (mCRC): Efficacy and tumor markers. American Society of Clinical Oncology, 2018.

20. Deming DA, Cavalcante LL, Lubner SJ, et al. A phase I study of selumetinib (AZD6244/ARRY-142866), a MEK1/2 inhibitor, in combination with cetuximab in refractory solid tumors and KRAS mutant colorectal

Cite this article as: Kratz JD, Deming DA. The evolving treatment paradigm for BRAF V600 mutant colorectal cancer. Ann Transl Med 2019;7(Suppl 8):S257. doi: 10.21037/ atm.2019.12.61 cancer. Investigational new drugs 2016;34:168-75.

21. Kopetz S, Grothey A, Yaeger R, et al. Encorafenib, binimetinib, and cetuximab in BRAF V600E-mutated colorectal cancer. N Engl J Med 2019;381:1632-43.

22. Jones JC, Renfro LA, Al-Shamsi HO, et al. NonV600BRAF mutations define a clinically distinct molecular subtype of metastatic colorectal cancer. J Clin Oncol 2017;35:2624-30.

23. Johnson B, Loree JM, Jacome AA, et al. Atypical, Non-V600 BRAF Mutations as a Potential Mechanism of Resistance to EGFR Inhibition in Metastatic Colorectal Cancer. JCO Precision Oncology 2019:1-10.

24. English DR, Young JP, Simpson JA, et al. Ethnicity and risk for colorectal cancers showing somatic BRAF V600E mutation or $\mathrm{CpG}$ island methylator phenotype. Cancer Epidemiol Biomarkers Prev 2008;17:1774-80.

25. Overman MJ, Lonardi S, Wong KYM, et al. Durable Clinical Benefit With Nivolumab Plus Ipilimumab in DNA Mismatch Repair-Deficient/Microsatellite Instability-High Metastatic Colorectal Cancer. J Clin Oncol 2018;36:773-9. 